\title{
Synthesis and Molecular Docking Studies of New Dispiropyrrolidines on West Nile Virus NS2B-NS3 Protease
}

\author{
Nadia Mohamed Yusoff ${ }^{1}$, Hasnah Osman ${ }^{1}$, Mohd. Zaheen Hassan ${ }^{1,2}$, Mohamed Ashraf Alii,3, Yeong \\ Keng Yoon ${ }^{4,5}$, Ezatul Ezleen Kamarulzaman ${ }^{6}$, Muhammad Solehin Abd Ghani ${ }^{1}$, Unang Supratman ${ }^{7}$, \\ and Mohamad Nurul Azmi Mohamad Taib ${ }^{1^{*}}$ \\ ${ }^{1}$ School of Chemical Sciences, Universiti Sains Malaysia, Minden 11800 Penang, Malaysia \\ ${ }^{2}$ College of Pharmacy, King Khalid University, Abha, Saudi Arabia \\ ${ }^{3}$ Department of Medicinal Chemistry, Sunrise University Alwar, Rajasthan-301030, India \\ ${ }^{4}$ Institute for Research in Molecular Medicine, Universiti Sains Malaysia, Minden 11800 Penang, Malaysia \\ ${ }^{5}$ School of Science, Monash University Malaysia Campus, Bandar Sunway, 47500, Subang Jaya, Selangor, Malaysia \\ ${ }^{6}$ School of Pharmaceutical Sciences, Universiti Sains Malaysia, Minden 11800 Penang, Malaysia \\ ${ }^{7}$ Department of Chemistry, Faculty of Mathematics and Natural Sciences, Universitas Padjadjaran, \\ Jl. Raya Bandung-Sumedang Km. 21, Jatinangor, Sumedang 45363, Indonesia
}

\section{* Corresponding author:}

email:mnazmi@usm.my

Received: May 21, 2021

Accepted: August 13, 2021

DOI: $10.22146 / \mathrm{ijc} .66017$

\begin{abstract}
West Nile virus (WNV) is among the other four flavivirus genus, rapidly spreading worldwide. The number of cases increases globally as there are no clinically available approved drugs and vaccines against this disease. Based on our previous finding related to a flavivirus, a series of spiropyrrolidine derivatives were regioselectively synthesized via [3+2]-cycloaddition reaction of three components between isatins, sarcosine, and (E)-3,5-bis (arylidene)-4-piperidones. The yield of synthesized compounds was in a range between 81-95\%. The structures of all the synthesized compounds were characterized using FT-IR, $1 D$ - and 2D-NMR, and HRMS. Molecular docking studies of spiropyrrolidines on NS2B-NS3 protease were done to understand and explore the ligandreceptor interactions and hypothesize the drug's refinements. The inhibition of NS2B-NS3 protease has been considered a promising strategy because this enzyme is responsible for the viral replication process. Among them, compound $5 c$ shows an excellent binding affinity with $-7.71 \mathrm{kcal} / \mathrm{mol}$ free binding energy and an inhibition constant of $1.73 \mu \mathrm{M}$. It also showed the binding orientation into the active site of WNV NS2B-NS3 protease on Asn84, Tyr1161, Gly1151, and Gly1153.
\end{abstract}

Keywords: spiropyrrolidine; [3+2]-cycloaddition; molecular docking; West Nile virus; WNV NS2B-NS3 protease

\section{- INTRODUCTION}

The flaviviral disease is an infectious disease transmitted by mosquitoes. The members of the Flavivirus genus include dengue virus (DENV), West Nile virus (WNV), yellow fever virus (YFV), and Japanese encephalitis (JEV) [1-3]. The number of cases increases day by day worldwide [1-3]. Thus, there is a pressing need to develop newer agents against these viruses. Discovered in 1937 in the West Nile district of Uganda, WNV was first isolated from the blood of a woman with mild febrile infection [4-5]. Since then, primary and sporadic outbreaks have been commonly recorded in Africa, Middle East, and Europe in the 1960s [4-5]. Infections in humans are typically asymptomatic or cause a moderate flu-like disease called West Nile fever for a few days. Recent WNV infections have been associated with much 
higher fatality rates, especially among the elderly [6-8].

WNV is transmitted to humans by the bites of infected mosquitoes of several species, including Culex sp., Aedes sp., and Anopheles sp. [9]. Symptomatic cases cause headaches, fever, drowsiness, lethargy, dizziness, nausea, and confusion, escalating to encephalitis, convulsions, seizures, and eventually death. Among all arthropod-borne Flaviviruses, WNV has the broadest geographic distribution and the most diverse vector and host variety, increasing its potential as a global health threat [9]. In 2020, there were 315 human cases of WNV infection in Mediterranean countries reported by the European Centre for Disease Prevention and Control (ECDC). Greece and Spain reported the highest cases with 143 and 77, respectively [10]. In WNV, an NS3 protease is associated with NS2B, forming a complex enzymatic NS2B-NS3, which is important for synthesizing the polyprotein precursor in viral replication [11-12]. Hence, NS2B-NS3 protease has been considered a favorable target to prevent virus infection and lead to flaviviral death [11,13-15]. Most of the research for flaviviral infections focuses on the inhibition of the NS2B-NS3 protease. In addition, the complexity of this enzyme is very challenging due to its shallow and open pocket active site [16].

Previous studies reported considerable biological activities of the dispiropyrrolidines on antimycobacterial [17-20], antifungal [21], antimicrobial [22-25], antitumor [26-28], anti-neoplastic [29], and antidiabetic activities [30-31]. No reported data explained the activity and interaction between dispiropyrrolidines and their roles as WNV NS2B-NS3 protease inhibitors. Previously, we reported the synthesis of new dibenzylidene-1phenylethylpiperidine-4-ones and new dispiropyrrolidines to evaluate their application as antimycobacterial against Mycobacterium tuberculosis [17-19,32]. Based on our previous achievement and our continuation on this research domain, this manuscript focuses on synthesizing new dispiropyrrolidines as a WNV NS2B-NS3 protease inhibitor, which is the recent finding dibenzylidene-1phenylethylpiperidine-4-ones are employed as dipolarophiles in [3+2]-cycloaddition reactions of azomethine ylide for the synthesis of new dispiropyrrolidines. The dispiropyrrolidines were investigated as potential WNV NS2B-NS3 protease inhibitors using a molecular docking approach. This information is very useful for predicting the binding behavior in the rational design of drugs and elucidating the fundamentals of biochemical processes.

\section{- EXPERIMENTAL SECTION}

\section{Materials}

Unless otherwise noted, materials were purchased from Sigma-Aldrich Co., Acros Organics, QReC, and Merck Chemical Co., i.e., 1-phenylethyl-4-piperidone 98\%, Benzaldehyde 99\%, 4-Methoxybenzaldehyde $98 \%$, 4-Methylbenzaldehyde 97\%, 4-Methoxybenzaldehyde 97\%, Isatin 98\%, 5-Chloroisatin 95\%, Sarcosine 98\%, Acetone, AR Grade, Ethanol, AR Grade, Methanol, AR Grade, Hexane AR Grade and Methyl sulfoxide- $\mathrm{d}_{6-}$ deuteration degree for NMR. All chemicals and solvents were of reagent grade and were used without further purification. Column chromatography was performed using Merck silica gel $(40-63 \mu \mathrm{m})$.

\section{Instrumentation}

Thin-layer chromatography (TLC) was performed on alumina plates pre-coated with silica gel (Merck silica gel, 60 F254), which were visualized by the quenching of UV fluorescence when applicable ( $\lambda_{\max }=254 \mathrm{~nm}$ and/or $366 \mathrm{~nm}$ ) and/or by spraying with vanillin or anisaldehyde in acidic ethanol followed by heating with a heat gun. It was performed using a solvent system of benzenemethanol (8:2) and toluene-ethyl formate-formic acid (5:4:1) to check the reactions' completion. All reactions were carried out in heat-dried glassware under a dry nitrogen atmosphere unless otherwise stated. All liquids transfer was conducted using standard syringe or cannula techniques. All spectral data were obtained on the following instruments: Infrared spectra were recorded on a Perkin Elmer 2000 FTIR spectrometer at wavenumber from $4000-600 \mathrm{~cm}^{-1} .{ }^{1} \mathrm{H}(500 \mathrm{MHz})$ and ${ }^{13} \mathrm{C}$ $(125 \mathrm{MHz})$ Nuclear magnetic resonance spectra were obtained on Bruker AVN $500 \mathrm{MHz}$ spectrometers (Bruker Bioscience, Billerica, MA, USA) which were reported in units of ppm on the $\delta$ scale. NMR analyses were done using solvent DMSO- $\mathrm{d}_{6}$ and TMS as the 
internal standard. Data were analyzed via the TopSpin software package. The chemical shift was internally referenced to the solvent signals in DMSO- $\mathrm{d}_{6}\left({ }^{1} \mathrm{H} \delta 2.50\right.$; $\left.{ }^{13} \mathrm{C} \delta 39.5\right)$. The coupling constants are given in $\mathrm{Hz}$. The mass spectra were measured using the Waters Xevo QTOF MS system.

\section{Procedure}

\section{Synthesis of (3E,5E)-3,5-bis(substitutedarylidene)-1- phenethyl piperidin-4-one (1a-c)}

The procedure of preparation of dibenzylidene-1phenylethylpiperidine-4-ones 1a-c was reported previously by our group [32]. 1-phenylethyl-4-piperidone (1.0 equiv.) and appropriate aldehyde (2.0 equiv.) were dissolved together in ethanol $(10 \mathrm{~mL})$ and $30 \%$ sodium hydroxide $(5 \mathrm{~mL}$; prepared in ethanol). The reaction mixture was stirred for 2-6 h at room temperature until completion (TLC). The mixture was then poured into crushed ice. The precipitated solid was filtered, washed with water, and purified by recrystallization. The spectroscopic data were compared with the literature [32].

\section{(3E,5E)-3,5-dibenzylidene-1-phenylethyl-4-}

piperidinone (1a). 1-Phenylethyl-4-piperidone (1.22 g, $6.0 \mathrm{mmol})$ and benzaldehyde $(1.27 \mathrm{~g}, 12.0 \mathrm{mmol})$ were dissolved together in ethanol $(10 \mathrm{~mL})$ and $30 \%$ sodium hydroxide ( $5 \mathrm{~mL}$; prepared in ethanol) according to the general procedure above. The precipitated solid was filtered, washed with water and purified by recrystallization to give a bright yellow solid (2.12 g, 94.6\%), M.p. $167-170^{\circ} \mathrm{C}$. FTIR (ATR, $\mathrm{cm}^{-1}$ ): 3026 (w, C$\mathrm{H}), 1669$ (s, C=O), 1608 (s, C=C), $1181(\mathrm{~m}, \mathrm{C}-\mathrm{N}) .{ }^{1} \mathrm{H}-$ NMR (500 MHz, DMSO-d $\left.\mathrm{d}_{6}\right): \delta_{\mathrm{H}}, \mathrm{ppm} 2.70(2 \mathrm{H}, \mathrm{t}, J=7.5$ $\left.\mathrm{Hz}, 8-\mathrm{CH}_{2}\right), 2.81\left(2 \mathrm{H}, \mathrm{t}, J=7.5 \mathrm{~Hz}, 7-\mathrm{CH}_{2}\right), 3.88(4 \mathrm{H}, \mathrm{s}, 2-$ $\mathrm{CH}_{2}, 6-\mathrm{CH}_{2}$ ), 7.14-7.23 (5H, m, H-10, H-11, H-12, H-13, $\mathrm{H}-14), 7.42-7.51(10 \mathrm{H}, \mathrm{m}, \mathrm{H}-17, \mathrm{H}-18, \mathrm{H}-19, \mathrm{H}-20, \mathrm{H}-$ 21), 7.58 (2H, s, H-15). ${ }^{13} \mathrm{C}-\mathrm{NMR}\left(125 \mathrm{MHz}, \mathrm{DMSO}-\mathrm{d}_{6}\right)$ : $\delta_{\mathrm{C}}, \mathrm{ppm} 33.3,54.5,58.3,126.3,128.6,129.0,129.2,129.7$, 131.0, 134.3, 135.1, 135.3, 140.5, 187.4. HRMS (TOF-ES ${ }^{+}$): $\mathrm{m} / \mathrm{z} 380.2139\left(\mathrm{MH}^{+} \mathrm{C}_{27} \mathrm{H}_{26} \mathrm{NO}^{+}\right.$requires 380.2009).

(3E,5E)-3,5-bis(4-methylbenzylidene)-1-phenylethyl4-piperidinone (1b). 1-Phenylethyl-4-piperidone (1.22 g, $6.0 \mathrm{mmol}$ ) and 4-methylbenzaldehyde (1.44 g, $12.0 \mathrm{mmol}$ ) were dissolved together in ethanol $(10 \mathrm{~mL})$ and $30 \%$ sodium hydroxide ( $5 \mathrm{~mL}$; prepared in ethanol) according to the general procedure above. The precipitated solid was filtered, washed with water and purified by recrystallization to give a yellow solid $(2.19 \mathrm{~g}, 90.1 \%)$, M.p. $118-121^{\circ} \mathrm{C}$. FTIR (ATR, $\left.\mathrm{cm}^{-1}\right): 3024(\mathrm{w}, \mathrm{C}-\mathrm{H})$, 1668 (s, C=O), 1606 (m, C=C), $1174(\mathrm{~m}, \mathrm{C}-\mathrm{N}) .{ }^{1} \mathrm{H}-\mathrm{NMR}$ $\left(500 \mathrm{MHz}, \mathrm{DMSO}-\mathrm{d}_{6}\right): \delta_{\mathrm{H}}, \mathrm{ppm} 2.36\left(6 \mathrm{H}, \mathrm{s}, 22-\mathrm{CH}_{3}\right)$, $2.71\left(2 \mathrm{H}, \mathrm{t}, J=7.5 \mathrm{~Hz}, 8-\mathrm{CH}_{2}\right), 2.81(2 \mathrm{H}, \mathrm{t}, J=7.5 \mathrm{~Hz}, 7-$ $\left.\mathrm{CH}_{2}\right), 3.86\left(4 \mathrm{H}, \mathrm{s}, 2-\mathrm{CH}_{2}, 6-\mathrm{CH}_{2}\right), 7.14-7.24(5 \mathrm{H}, \mathrm{m}, \mathrm{H}-$ $10, \mathrm{H}-11, \mathrm{H}-12, \mathrm{H}-13, \mathrm{H}-14), 7.29(4 \mathrm{H}, \mathrm{d}, J=8.5 \mathrm{~Hz}, \mathrm{H}-$ 18, H-20), 7.40 (4H, d, J = $8.50 \mathrm{~Hz}, \mathrm{H}-17, \mathrm{H}-21,), 7.58$ $(2 \mathrm{H}, \mathrm{s}, \mathrm{H}-15) .{ }^{13} \mathrm{C}-\mathrm{NMR}\left(125 \mathrm{MHz}, \mathrm{DMSO}-\mathrm{d}_{6}\right): \delta_{\mathrm{c}}, \mathrm{ppm}$ 21.5, 33.3, 54.6, 58.4, 126.3, 128.7, 129.0, 129.9, 131.0, $132.4,133.5,135.2,139.6,140.5,187.3$. HRMS (TOF$\left.\mathrm{ES}^{+}\right): \mathrm{m} / \mathrm{z} 408.2324\left(\mathrm{MH}^{+} \mathrm{C}_{29} \mathrm{H}_{30} \mathrm{NO}^{+}\right.$requires 408.2322).

\section{(3E,5E)-3,5-bis(4-methoxybenzylidene)-1-} phenylethyl-4-piperidinone (1c). 1-Phenylethyl-4piperidone $(0.61 \mathrm{~g}, \quad 3.0 \mathrm{mmol})$ and 4 methoxybenzaldehyde $(0.82 \mathrm{~g}, 6.0 \mathrm{mmol})$ were dissolved together in ethanol $(5 \mathrm{~mL})$ and $30 \%$ sodium hydroxide ( $3 \mathrm{~mL}$; prepared in ethanol) according to the general procedure above. The precipitated solid was filtered, washed with water and purified by recrystallization to give a yellow solid (1.13 g, 85.4\%), M.p. $143-146^{\circ} \mathrm{C}$, Lit. M.p. $147^{\circ} \mathrm{C}$. FTIR (ATR, $\mathrm{cm}^{-1}$ ): 3023 (w, C-H), 1667 (s, $\mathrm{C}=\mathrm{O}), 1596(\mathrm{~m}, \mathrm{C}=\mathrm{C}), 1166(\mathrm{~m}, \mathrm{C}-\mathrm{N}) ;{ }^{1} \mathrm{H}-\mathrm{NMR}(500$ MHz, DMSO-d $\left.{ }_{6}\right): \delta_{\mathrm{H}}, \mathrm{ppm} 2.73(2 \mathrm{H}, \mathrm{t}, J=7.5 \mathrm{~Hz} 8-$ $\left.\mathrm{CH}_{2}\right), 2.82\left(2 \mathrm{H}, \mathrm{t}, J=7.5 \mathrm{~Hz}, 7-\mathrm{CH}_{2}\right), 3.82(6 \mathrm{H}, \mathrm{s}, 22-$ $\left.\mathrm{OCH}_{3}\right), 3.84\left(4 \mathrm{H}, \mathrm{s}, 2-\mathrm{CH}_{2}, 6-\mathrm{CH}_{2}\right), 7.04(4 \mathrm{H}, \mathrm{d}, J=8.5$ $\mathrm{Hz}, 18-\mathrm{CH}, 20-\mathrm{CH}$ ), 7.15-7.25 (5H, m, 10-CH, 11-CH, 12- $\mathrm{CH}, 13-\mathrm{CH}, 14-\mathrm{CH}), 7.45(4 \mathrm{H}, \mathrm{d}, J=8.5 \mathrm{~Hz}, 17-\mathrm{CH}$, 21-CH), 7.56 (2H, s, H-15). ${ }^{13} \mathrm{C}-\mathrm{NMR}(125 \mathrm{MHz}$, DMSO-d $\left.{ }_{6}\right): \delta_{\mathrm{C}}, \mathrm{ppm} 33.3,54.7,55.8,58.5,113.7,114.8$, $126.3,127.7,128.7,129.0,132.2,132.9,134.9,140.6$, 160.5, 187.1. HRMS (TOF-ES $\left.{ }^{+}\right): \mathrm{m} / \mathrm{z} 440.2399\left(\mathrm{MH}^{+}\right.$ $\mathrm{C}_{29} \mathrm{H}_{30} \mathrm{NO}_{3}{ }^{+}$requires 440.2226).

\section{Synthesis of spiropyrrolidines 5 and 6}

3,5-Bis(substitutedarylidene)-1-phenethylpiperidin4-one (1) (1.0 equiv.) was refluxed together with appropriate isatins 3 or 4 (1.0 equiv.) and sarcosine (2) (2.0 equiv.) in methanol $(20 \mathrm{~mL})$ for $4-7 \mathrm{~h}$. After completing the reaction, the excess solvent in the mixture was removed under reduced pressure and cooled before being poured onto crushed ice. The 
products were filtered, washed with water, and purified by recrystallization.

1-Methyl-4(phenyl)pyrrolo-(spiro[2.3"']oxindole)spiro[3.3']-5'-(phenylmethylidene)-1'-phenylethyl4'-piperidinone (5a). 3,5-Bis(substitutedarylidene)-1phenethylpiperidin-4-one 1a $(0.10 \mathrm{~g}, 0.26 \mathrm{mmol})$ was refluxed together with appropriate isatin $3(0.04 \mathrm{~g}, 0.26$ $\mathrm{mmol})$ and sarcosine (2) $(0.05 \mathrm{~g}, 0.52 \mathrm{mmol})$ in methanol $(20 \mathrm{~mL})$ according to the general procedure above. Products obtained were filtered, washed with water and purified by recrystallization to give pale yellow solid (Yield $=0.13 \mathrm{~g}(89.6 \%)$, M.p. $117-120^{\circ} \mathrm{C}$. FTIR $\left(\right.$ ATR $\left.\mathrm{cm}^{-1}\right): v$ 3346 (w, N-H), 3027 (w, C-H), 1697 (s, C=O), 1599 (m, $\mathrm{C}=\mathrm{C}), 1182(\mathrm{~m}, \mathrm{C}-\mathrm{N}) .{ }^{1} \mathrm{H}-\mathrm{NMR}\left(500 \mathrm{MHz}, \mathrm{DMSO}-\mathrm{d}_{6}\right)$ : $\delta_{\mathrm{H}}, \mathrm{ppm}: 1.76\left(1 \mathrm{H}, \mathrm{d}, J=12.5 \mathrm{~Hz}, 7^{\prime}-\mathrm{CH}_{2 \mathrm{a}}\right), 2.00(\mathrm{~s}, 3 \mathrm{H}, N-$ $\left.\mathrm{CH}_{3}\right), 2.34-2.45\left(\mathrm{~m}, 4 \mathrm{H}, 2^{\prime}-\mathrm{CH}_{2}, 6^{\prime}-\mathrm{CH}_{2}\right), 3.06(\mathrm{dd}, 1 \mathrm{H}, J=$ 2.4, $\left.2.5 \mathrm{~Hz}, 5-\mathrm{CH}_{2 \mathrm{a}}\right), 3.20-3.25\left(\mathrm{~m}, 2 \mathrm{H}, 7^{\prime}-\mathrm{CH}_{2 \mathrm{~b}}, 8^{\prime}-\mathrm{CH}_{2 \mathrm{a}}\right)$, 3.36 ( ${ }^{*}$ overlap signal, $\left.1 \mathrm{H}, 5-\mathrm{CH}_{2 \mathrm{~b}}\right), 3.82-3.86\left(\mathrm{~m}, 1 \mathrm{H}, 8^{\prime}-\right.$ $\mathrm{CH}_{2 \mathrm{a}}$ ), 4.64-4.68 (m, 1H, H-4), 6.65-7.33 (19H, m, ArH), $10.46(\mathrm{~s}, 1 \mathrm{H}, \mathrm{NH}) .{ }^{13} \mathrm{C}-\mathrm{NMR}\left(125 \mathrm{MHz}, \mathrm{DMSO}-\mathrm{d}_{6}\right) \delta_{\mathrm{C}}$, ppm: 32.0, 34.1, 45.2, 53.9, 56.2, 56.3, 59.1, 64.6, 75.2, $108.7,120.7,125.8,126.75,126.85,126.91,128.18,128.20$, $128.46,128.47,128.6,128.9,129.1,129.1,130.0,132.9$, $134.5,136.6,138.4,140.0,143.4,176.6,198.2$. HRMS (TOF-ES $)^{+}: \mathrm{m} / \mathrm{z} 554.2899\left(\mathrm{MH}^{+} \mathrm{C}_{37} \mathrm{H}_{36} \mathrm{~N}_{3} \mathrm{O}_{2}{ }^{+}\right.$requires 554.2803).

\section{1-Methyl-4(-4-methylphenyl)pyrrolo-} (spiro[2.3']oxindole)-spiro[3.3']-5'-(4methylphenylmethylidene)-1'-phenylethyl-4'-

piperidinone (5b). 3,5-Bis(substitutedarylidene)-1phenethylpiperidin-4-one $\mathbf{1 b}(0.10 \mathrm{~g}, 0.26 \mathrm{mmol})$ was refluxed together with appropriate isatin $3(0.04 \mathrm{~g}, 0.26$ $\mathrm{mmol}$ ) and sarcosine (2) (0.05 g, $0.52 \mathrm{mmol})$ in methanol $(20 \mathrm{~mL})$ according to the general procedure above. Products obtained were filtered, washed with water and purified by recrystallization to give pale yellow solid (Yield $=0.13 \mathrm{~g}(88.7 \%)$, M.p. $192-195^{\circ} \mathrm{C}$. FTIR $\left(\right.$ ATR, $\left.\mathrm{cm}^{-1}\right): v$ 3397 (w, N-H), 3023 (w, C-H), 1694 (s, C=O, 1605 (m, $\mathrm{C}=\mathrm{C}), 1177(\mathrm{~m}, \mathrm{C}-\mathrm{N}) .{ }^{1} \mathrm{H}-\mathrm{NMR}\left(500 \mathrm{MHz}, \mathrm{DMSO}-\mathrm{d}_{6}\right)$ $\delta_{\mathrm{H}}, \mathrm{ppm}: 1.78\left(\mathrm{~d}, 1 \mathrm{H}, J=12.5 \mathrm{~Hz}, 7^{\prime}-\mathrm{CH}_{2 \mathrm{a}}\right), 1.98(\mathrm{~s}, 3 \mathrm{H}, \mathrm{N}-$ $\left.\mathrm{CH}_{3}\right), 2.27\left(\mathrm{~d}, 6 \mathrm{H}, J=5.8 \mathrm{~Hz}, 2 \times \mathrm{CH}_{3}\right), 2.33-2.46(\mathrm{~m}, 4 \mathrm{H}$, $\left.2^{\prime}-\mathrm{CH}_{2}, 6^{\prime}-\mathrm{CH}_{2}\right), 3.05\left(\mathrm{dd}, 1 \mathrm{H}, J=2.4,2.5 \mathrm{~Hz}, 5-\mathrm{CH}_{2 \mathrm{a}}\right.$ ), 3.17-3.22 ( $\left.\mathrm{m}, 2 \mathrm{H}, 7^{\prime}-\mathrm{CH}_{2 \mathrm{~b}}, 8^{\prime}-\mathrm{CH}_{2 \mathrm{a}}\right)$, $3.26{ }^{*}$ overlap signal,
$1 \mathrm{H}, 5-\mathrm{CH}_{2 \mathrm{~b}}$ ), 3.78-3.82 (m, $\left.1 \mathrm{H}, 8^{\prime}-\mathrm{CH}_{2 \mathrm{~b}}\right), 4.58(\mathrm{dd}, 1 \mathrm{H}, \mathrm{J}$ $=7.4,7.5 \mathrm{~Hz}, \mathrm{H}-4), 6.63-7.26(18 \mathrm{H}, \mathrm{m}, \mathrm{ArH}), 10.36(\mathrm{~s}$, $1 \mathrm{H}, \mathrm{NH}) .{ }^{13} \mathrm{C}-\mathrm{NMR}\left(125 \mathrm{MHz}, \mathrm{DMSO}-\mathrm{d}_{6}\right) \delta_{\mathrm{C}}, \mathrm{ppm}: 20.6$, 20.9, 32.1, 34.1, 44.8, 54.0, 56.3, 56.4, 59.1, 64.5, 75.2, $108.6,120.6,125.8,126.88,126.93,128.2,128.5,128.4$, $128.8,129.0,129.1,130.2,131.7,132.1,135.3,135.7$, 136.6, 138.8, 140.1, 143.4, 176.6, 198.2. HRMS (TOF$\left.\mathrm{ES}^{+}\right): \quad \mathrm{m} / \mathrm{z} \quad 582.3081 \quad\left(\mathrm{MH}^{+} \quad \mathrm{C}_{39} \mathrm{H}_{40} \mathrm{~N}_{3} \mathrm{O}_{2}{ }^{+}\right.$requires $582.3115,604.2894 \quad\left(\mathrm{MNa}^{+} \mathrm{C}_{39} \mathrm{H}_{39} \mathrm{~N}_{3} \mathrm{NaO}_{2}{ }^{+}\right.$requires 604.2935).

\section{1-Methyl-4(-4-methoxyphenyl)pyrrolo-} (spiro[2.3']oxindole)-spiro[3.3']-5'-(4methoxyphenylmethylidene)-1'-phenylethyl-4'piperidinone (5c). 3,5-Bis(substitutedarylidene)-1phenethylpiperidin-4-one $1 \mathrm{c}(0.10 \mathrm{~g}, 0.26 \mathrm{mmol})$ was refluxed together with appropriate isatin $3(0.04 \mathrm{~g}$, $0.26 \mathrm{mmol})$ and sarcosine (2) (0.05 g, $0.52 \mathrm{mmol})$ in methanol $(20 \mathrm{~mL})$ according to the general procedure above. Products obtained were filtered, washed with water and purified by recrystallization to give pale yellow solid (Yield $=0.13 \mathrm{~g}$ (88.9\%), M.p. $130-133{ }^{\circ} \mathrm{C}$. FTIR $\left(\mathrm{ATR}, \mathrm{cm}^{-1}\right): 3289(\mathrm{w}, \mathrm{N}-\mathrm{H}), 2934(\mathrm{w}, \mathrm{C}-\mathrm{H}), 1706$ (s, $\mathrm{C}=\mathrm{O}), 1580(\mathrm{~m}, \mathrm{C}=\mathrm{C}), 1247$ (m, C-O), $1173(\mathrm{~m}, \mathrm{C}-\mathrm{N})$. ${ }^{1} \mathrm{H}-\mathrm{NMR}\left(500 \mathrm{MHz}, \mathrm{DMSO}-\mathrm{d}_{6}\right): \delta_{\mathrm{H}}, \mathrm{ppm} 1.76(1 \mathrm{H}, \mathrm{d}, J$ $\left.=12.5 \mathrm{~Hz}, 7^{\prime}-\mathrm{CH}_{2 \mathrm{a}}\right), 1.97\left(3 \mathrm{H}, \mathrm{s}, \mathrm{N}-\mathrm{CH}_{3}\right), 2.31-2.46(4 \mathrm{H}$, $\left.\mathrm{m}, 2^{\prime}-\mathrm{CH}_{2}, 6^{\prime}-\mathrm{CH}_{2}\right), 3.04\left(1 \mathrm{H}, \mathrm{dd}, J=2.3,2.4 \mathrm{~Hz}, 5-\mathrm{CH}_{2 \mathrm{a}}\right)$, 3.16-3.19 (2H, m, 7'- $\left.\mathrm{CH}_{2 \mathrm{~b}}, 8^{\prime}-\mathrm{CH}_{2 \mathrm{a}}\right), 3.40\left(1 \mathrm{H}\right.$, ${ }^{*}$ overlap signal, 5- $\left.\mathrm{CH}_{2 \mathrm{~b}}\right), 3.72\left(3 \mathrm{H}, \mathrm{s}, \mathrm{OCH}_{3}\right), 3.76\left(1 \mathrm{H}\right.$, ${ }^{*}$ overlap signal, $\left.8^{\prime}-\mathrm{CH}_{2 \mathrm{~b}}\right), 3.82\left(3 \mathrm{H}, \mathrm{s}, \mathrm{OCH}_{3}\right), 4.57(1 \mathrm{H}, \mathrm{dd}, J=7.5$, $7.5 \mathrm{~Hz}, \mathrm{H}-4), 6.64-7.26(20 \mathrm{H}, \mathrm{m}, \mathrm{ArH}), 10.38(1 \mathrm{H}, \mathrm{s}$, $\mathrm{NH}) .{ }^{13} \mathrm{C}-\mathrm{NMR}\left(125.8 \mathrm{MHz}, \mathrm{DMSO}-\mathrm{d}_{6}\right): \delta_{\mathrm{C}}, \mathrm{ppm} 32.6$, $34.5,45.0,54.7,55.4,55.7,55.8,56.7,57.1,59.8,64.8$, $75.9,109.1,121.1,126.3,127.4,127.5,127.6,127.7,128.7$, $128.9,129.0,130.6,130.8,131.2,132.2,132.9,132.9$, 137.0, 140.6, 143.9, 177.2, 198.6. HRMS (TOF-ES $\left.{ }^{+}\right): \mathrm{m} / \mathrm{z}$ $614.3027\left(\mathrm{MH}^{+} \mathrm{C}_{39} \mathrm{H}_{40} \mathrm{~N}_{3} \mathrm{O}_{4}{ }^{+}\right.$requires 614.3019).

1-Methyl-4(phenyl)pyrrolo-(spiro[2.3"]-5"chlorooxindole)-spiro[3.3']-5'(phenylmethylidene)-1'-phenylethyl-4'piperidinone (6a). 3,5-Bis(substitutedarylidene)-1phenethylpiperidin-4-one $1 \mathrm{a}(0.10 \mathrm{~g}, 0.26 \mathrm{mmol})$ was refluxed together with appropriate 5-chloroisatin $4(0.04$ g, $0.26 \mathrm{mmol})$ and sarcosine (2) $(0.05 \mathrm{~g}, 0.52 \mathrm{mmol})$ in 
methanol $(20 \mathrm{~mL})$ according to the general procedure above. Products obtained were filtered, washed with water and purified by recrystallization to give pale yellow solid (Yield $=0.15$ g (95.4\%), M.p. 131-134 ${ }^{\circ} \mathrm{C}$. FTIR (ATR, $\left.\mathrm{cm}^{-1}\right): v 3244(\mathrm{w}, \mathrm{N}-\mathrm{H}), 3027(\mathrm{w}, \mathrm{C}-\mathrm{H}), 1698(\mathrm{~s}, \mathrm{C}=\mathrm{O})$, $1615(\mathrm{~m}, \mathrm{C}=\mathrm{C}), 1182(\mathrm{~m}, \mathrm{C}-\mathrm{N}) .{ }^{1} \mathrm{H}-\mathrm{NMR}(500 \mathrm{MHz}$, DMSO-d $\left.{ }_{6}\right) \delta_{\mathrm{H}}, \mathrm{ppm}: 1.74-1.77\left(\mathrm{~d}, 1 \mathrm{H}, J=12.6 \mathrm{~Hz}, 7^{\prime}-\right.$ $\mathrm{CH}_{2 \mathrm{a}}$ ), 2.02 (s, 3H, N-CH $), 2.37-2.44\left(\mathrm{~m}, 4 \mathrm{H}, 2^{\prime}-\mathrm{CH}_{2}, 6^{\prime}-\right.$ $\left.\mathrm{CH}_{2}\right), 3.09-3.12\left(\mathrm{dd}, 1 \mathrm{H}, \mathrm{J}=2.4,2.5, \mathrm{~Hz}, 5-\mathrm{CH}_{2 \mathrm{a}}\right), 3.22-$ $3.25\left(\mathrm{~m}, 2 \mathrm{H}, 7^{\prime}-\mathrm{CH}_{2 \mathrm{~b}}, 8^{\prime}-\mathrm{CH}_{2 \mathrm{a}}\right), 3.37$ ( ${ }^{*}$ overlap signal, $1 \mathrm{H}$, 5- $\left.\mathrm{CH}_{2 \mathrm{~b}}\right), 3.80-3.84\left(1 \mathrm{H}, \mathrm{m}, 8^{\prime}-\mathrm{CH}_{2 \mathrm{~b}}\right), 4.63-4.67(\mathrm{~m}, 1 \mathrm{H}$, H-4), 6.66-6.83 (1H, m, ArH), 7.11-7.37 (19H, m, ArH), $10.57(\mathrm{~s}, 1 \mathrm{H}, \mathrm{NH}) .{ }^{13} \mathrm{C}-\mathrm{NMR}\left(125 \mathrm{MHz}, \mathrm{DMSO}-\mathrm{d}_{6}\right) \delta_{\mathrm{C}}$, ppm: 32.0, 34.2, 45.1, 54.0, 56.3, 56.4, 59.1, 65.1, 75.2, $110.3,125.0,125.9,126.8,126.9,128.27,128.34,128.5$, 128.6, 128.7, 129.17, 129.23, 129.1, 130.1, 133.1, 134.3, 137.2, 138.1, 140.0, 142.4, 176.3, 198.2. HRMS (TOF-ES ${ }^{+}$): $\mathrm{m} / \mathrm{z} 588.2390\left(\mathrm{MH}^{+} \mathrm{C}_{37} \mathrm{H}_{35}{ }^{35} \mathrm{ClN}_{3} \mathrm{O}_{2}{ }^{+}\right.$requires 588.2413).

\section{1-Methyl-4(-4-methylphenyl)pyrrolo-(spiro[2.3"]- \\ 5"-chlorooxindole)-spiro[3.3']-5'-(4- methylphenylmethylidene)-1'-phenylethyl-4'-}

piperidinone (6b). 3,5-Bis(substitutedarylidene)-1phenethyl piperidin-4-one $\mathbf{1 b}(0.10 \mathrm{~g}, 0.26 \mathrm{mmol})$ was refluxed together with appropriate 5-chloroisatin $4(0.04 \mathrm{~g}$, $0.26 \mathrm{mmol})$ and sarcosine (2) (0.05 g, $0.52 \mathrm{mmol})$ in methanol $(20 \mathrm{~mL})$ according to the general procedure above. Products obtained were filtered, washed with water and purified by recrystallization to give pale yellow solid (Yield $=0.13 \mathrm{~g}(84.4 \%)$, M.p. $186-189^{\circ} \mathrm{C}$. FTIR (ATR, $\mathrm{cm}^{-}$ $\left.{ }^{1}\right): v 3180(\mathrm{w}, \mathrm{N}-\mathrm{H}), 3025(\mathrm{w}, \mathrm{C}-\mathrm{H}), 1691(\mathrm{~s}, \mathrm{C}=\mathrm{O}), 1607$ (m, C=C), $1182(\mathrm{~m}, \mathrm{C}-\mathrm{N}) .{ }^{1} \mathrm{H}-\mathrm{NMR}(500 \mathrm{MHz}, \mathrm{DMSO}-$ $\left.\mathrm{d}_{6}\right) \delta_{\mathrm{H}}, \mathrm{ppm}: 1.75\left(\mathrm{~d}, 1 \mathrm{H}, J=12.5 \mathrm{~Hz}, 7^{\prime}-\mathrm{CH}_{2 \mathrm{a}}\right), 2.01(\mathrm{~s}, 3 \mathrm{H}$, $\left.\mathrm{N}-\mathrm{CH}_{3}\right), 2.30\left(\mathrm{~d}, 6 \mathrm{H}, J=11.6 \mathrm{~Hz}, 2 \times \mathrm{CH}_{3}\right), 2.36\left(\mathrm{~s}, 2 \mathrm{H}, 6^{\prime}-\right.$ $\mathrm{CH}_{2}$ ), 2.42-2.46 (m, $2 \mathrm{H}, 2^{\prime}-\mathrm{CH}_{2}$ ), 3.08 (dd, $1 \mathrm{H}, J=1.8,1.9$ $\mathrm{Hz}, 5-\mathrm{CH}_{2 \mathrm{a}}$ ), 3.17-3.22 (m, 2H, 7'- $\left.\mathrm{CH}_{2 \mathrm{~b}}, 8^{\prime}-\mathrm{CH}_{2 \mathrm{~b}}\right), 3.39$ ( ${ }^{*}$ overlap signal, $1 \mathrm{H}, 5-\mathrm{CH}_{2 \mathrm{~b}}$ ), $3.76-3.80\left(\mathrm{~m}, 1 \mathrm{H}, 8^{\prime}-\mathrm{CH}_{2 \mathrm{~b}}\right.$ ), $4.60(\mathrm{dd}, 1 \mathrm{H}, J=7.4,7.4 \mathrm{~Hz}, \mathrm{H}-4), 6.64-7.40(19 \mathrm{H}, \mathrm{m}$, $\mathrm{ArH}), 10.54$ (s, $1 \mathrm{H}, \mathrm{NH}) .{ }^{13} \mathrm{C}-\mathrm{NMR}\left(125 \mathrm{MHz}, \mathrm{DMSO}-\mathrm{d}_{6}\right)$ $\delta_{\mathrm{C}}$, ppm: 20.6, 20.9, 32.0, 34.1, 44.6, 54.0, 56.3, 56.4, 59.0, $64.9,75.2,110.1,124.8,125.8,126.8,128.2,128.3,128.4$, 128.8, 128.9, 129.1, 129.2, 130.1, 131.5, 132.2, 135.0, 135.8, 137.1, 139.1, 139.9, 142.3, 176.2, 198.1. HRMS (TOF-ES ${ }^{+}$): $\mathrm{m} / \mathrm{z}$ 616.2735 $\left(\mathrm{MH}^{+} \mathrm{C}_{39} \mathrm{H}_{39}{ }^{35} \mathrm{ClN}_{3} \mathrm{O}_{2}{ }^{+}\right.$requires 616.2726), $638.2595\left(\mathrm{MNa}^{+} \mathrm{C}_{39} \mathrm{H}_{38}{ }^{35} \mathrm{ClN}_{3} \mathrm{O}_{2} \mathrm{Na}^{+}\right.$requires 638.2545).

\section{1-Methyl-4(-4-methoxyphenyl)pyrrolo-} (spiro[2.3"]-5"-chlorooxindole)-spiro[3.3']-5'-(-4methoxyphenylmethylidene)-1'-phenylethyl-4'piperidinone (6c). 3,5-Bis(substitutedarylidene)-1phenethylpiperidin-4-one $1 \mathrm{c}(0.10 \mathrm{~g}, 0.26 \mathrm{mmol})$ was refluxed together with appropriate 5-chloroisatin 4 (0.04 g, $0.26 \mathrm{mmol})$ and sarcosine (2) $(0.05 \mathrm{~g}, 0.52 \mathrm{mmol})$ in methanol $(20 \mathrm{~mL})$ according to the general procedure above. Products obtained were filtered, washed with water and purified by recrystallization to give pale yellow solid (Yield $=0.11 \mathrm{~g}(81.3 \%)$, M.p. $187-190^{\circ} \mathrm{C}$. IR (ATR, $\left.\mathrm{cm}^{-1}\right)$ : 3297 (w, N-H), 2943 (w, C-H), 1709 (s, C=O), 1578 (m, C=C), $1250(\mathrm{C}-\mathrm{O}), 1172(\mathrm{C}-\mathrm{N}) .{ }^{1} \mathrm{H}-\mathrm{NMR}(500$ MHz, DMSO- $\left.\mathrm{d}_{6}\right): \delta_{\mathrm{H}}, \mathrm{ppm} 1.76\left(\mathrm{~d}, 1 \mathrm{H}, \mathrm{d}, J=12.5 \mathrm{~Hz}, 7^{\prime}-\right.$ $\mathrm{CH}_{2 \mathrm{a}}$ ), $2.00\left(\mathrm{~s}, 3 \mathrm{H}, \mathrm{N}-\mathrm{CH}_{3}\right), 2.37-2.43\left(\mathrm{~m}, 4 \mathrm{H}, 2^{\prime}-\mathrm{CH}_{2}, 6^{\prime}-\right.$ $\left.\mathrm{CH}_{2}\right), 3.10\left(\mathrm{dd}, 1 \mathrm{H}, J=2.4,2.5 \mathrm{~Hz}, 5-\mathrm{CH}_{2 \mathrm{a}}\right), 3.16-3.21$ $\left(\mathrm{m}, 2 \mathrm{H}, 7^{\prime}-\mathrm{CH}_{2 \mathrm{~b}}, 8^{\prime}-\mathrm{CH}_{2 \mathrm{a}}\right.$ ), 3.38 ( ${ }^{*}$ overlap signal, $1 \mathrm{H}, 5$ $\left.\mathrm{CH}_{2 \mathrm{~b}}\right), 3.73$ (s, $\left.3 \mathrm{H}, \mathrm{OCH}_{3}\right), 3.75$ ( ${ }^{*}$ overlap, $1 \mathrm{H}, 8^{\prime}-\mathrm{CH}_{2 \mathrm{~b}}$ ), $3.78\left(\mathrm{~s}, 3 \mathrm{H}, \mathrm{OCH}_{3}\right), 4.60(\mathrm{dd}, 1 \mathrm{H}, J=7.65,7.55 \mathrm{~Hz}, \mathrm{H}-4)$, 6.65-7.27 (17H, m, ArH), 10.55 (s, 1H, NH). ${ }^{13} \mathrm{C}-\mathrm{NMR}$ (125.8 MHz, DMSO-d $\mathrm{d}_{6}$ ): $\delta_{\mathrm{C}}, \mathrm{ppm} 32.5,34.6,44.8,54.7$, 55.4, 55.7, 56.8, 57.2, 59.7, 65.2, 75.8, 110.5, 124.8, 125.3, $126.3,128.3,128.4,128.7,128.8,128.9,129.7,130.5$, $130.6,131.3,132.7,135.1,135.9,137.5,139.0,140.5$, $142.8,176.8,198.5$. HRMS (TOF-ES ${ }^{+}$): $\mathrm{m} / \mathrm{z} 670.2453$ $\left(\mathrm{MNa}^{+} \mathrm{C}_{39} \mathrm{H}_{38} \mathrm{ClN}_{3} \mathrm{O}_{4} \mathrm{Na}^{+}\right.$requires 670.2449).

\section{Molecular docking studies}

Autodock 4.2 software was used to dock the ligands to the catalytic triad of NS3-NS2B protease. The target protein of West Nile virus (PDB: 2YOL) structure with a resolution of $3.20 \AA$ was retrieved from the RCSB Protein Data Bank. Water and chlorine atoms molecules were removed from the crystal structure. The native ligand, $\mathrm{EBN}$ in the $2 \mathrm{YOL}$, was extracted and re-docked with a grid box in a dimension of $40 \AA \times 40 \AA \times 40 \AA$ along the $\mathrm{x}, \mathrm{y}, \mathrm{z}$ coordinates to investigate the root mean square deviation ( $\mathrm{rmsd}$ ) between crystal geometry and the docked pose. The low rmsd value of 1.50-1.82 $\AA$ indicated that the docking was able to reproduce the native conformation. 


\section{- RESULTS AND DISCUSSION}

\section{Chemistry}

In the first step, we prepared the starting material of a dipolarophile known as dibenzylidene-1-phenylethyl piperidine-4-ones (1) via Claisen-Schmidt condensation between 1-phenylethyl-4-piperidone with an appropriate aromatic aldehyde in dilute ethanolic sodium hydroxide at room temperature. The completion of the reaction was checked using thin-layer chromatography (TLC) [32]. Next, we examined the three-component reaction of 1 and sarcosine (2) and isatin analogs ( 3 or 4 ) in methanol under reflux conditions to give the spiropyrrolidines ( 5 and 6), according to the method developed in our laboratory [19]. The reaction of spiropyrrolidine was conducted using two different isatin types: isatin (3) and 5-chloroisatin (4). The compounds 5 and $\mathbf{6}$ described in this study are shown in Scheme 1. The yield of synthesized compounds was in a range between 81-95\%.

The structures and regiochemistry of products 5-6 were characterized by IR, 1D- and 2D-NMR spectroscopy data, and HRMS analysis. Compound $\mathbf{6 b}$ is taken as an example to describe the result of analyses done. The FTIR spectrum of compound $\mathbf{6 b}$ manifests an absorption band at $3180 \mathrm{~cm}^{-1}$ attributed to the $\mathrm{N}-\mathrm{H}$ bending vibration, the aromatic band of $\mathrm{C}-\mathrm{H}$ stretch at $3025 \mathrm{~cm}^{-1}$, and $\mathrm{C}-\mathrm{N}$ stretching occur at $1182 \mathrm{~cm}^{-1}$. In addition, the aromatic $\mathrm{C}=\mathrm{C}$ stretching band can be observed at $1606 \mathrm{~cm}^{-1}$. The strong corresponding out of the plane C$\mathrm{H}$ bending vibration band appears at $820 \mathrm{~cm}^{-1}$, indicating a para-disubstituted ring in compound $\mathbf{6 b}$. The structure of dispiropyrrolidine $\mathbf{6 b}$ is agreed with the 1D- and 2D-NMR spectroscopic data. The ${ }^{1} \mathrm{H}-\mathrm{NMR}$ spectrum of $\mathbf{6 b}$ displayed two singlets at $\delta_{\mathrm{H}} 2.01$, and 10.54 was corresponding to the $\mathrm{N}-\mathrm{CH}_{3}$ and $\mathrm{N}-\mathrm{H}$, while the doublet at $\delta_{\mathrm{H}} 2.30(J=11.6 \mathrm{~Hz})$ was corresponding to $29^{\prime}-\mathrm{CH}_{3}$ and $28^{\prime}-\mathrm{CH}_{3}$, respectively. The doublet at $\delta_{\mathrm{H}} 1.75$ $(J=12.5 \mathrm{~Hz})$ and multiplet at $\delta_{\mathrm{H}} 2.42-2.46$ were assigned to $7^{\prime}-\mathrm{CH}_{2 \mathrm{a}}$ and $2^{\prime}-\mathrm{CH}_{2}$, respectively. The proton signal of 5- $\mathrm{CH}_{2 \mathrm{a}}$ and $\mathrm{H}-4$ appeared as doublets of a doublet at $\delta_{\mathrm{H}}$ $3.08(J=1.8,1.9 \mathrm{~Hz})$ and $4.60(J=7.4,7.4 \mathrm{~Hz})$.

The ${ }^{1} \mathrm{H}-{ }^{1} \mathrm{H}$-COSY spectrum of $\mathbf{6 b}$ revealed a cross peak between two neighboring protons of $\mathrm{H}_{-} \mathrm{7}^{\prime}$ and $\mathrm{H}$ $8^{\prime}$, while $\mathrm{H}-4$ showed a cross peak with its neighboring proton of $5-\mathrm{H}_{2 \mathrm{a}}$. Two multiplets at $\delta_{\mathrm{H}} 3.17-3.22$ and 3.76-3.80 were assigned to $7^{\prime}-\mathrm{CH}_{2 \mathrm{~b}} / 8^{\prime}-\mathrm{CH}_{2 \mathrm{~b}}$ and $8^{\prime}-\mathrm{CH}_{2 \mathrm{~b}}$, respectively. The presence of pyrrolidine ring attached at C- $3^{\prime}$ causes $\mathrm{H}-2^{\prime}$ to become more shielded than $\mathrm{H}-6^{\prime}$. Hence, the proton signal of $\mathrm{H}_{\mathrm{a}}-2^{\prime}$ is located at more upfield than $\mathrm{H}_{\mathrm{b}}-\mathbf{6}^{\prime}$ in $\mathbf{6} \mathbf{b}$. The multiplet proton signals that appeared in the aromatic region at $\delta_{\mathrm{H}} 6.64-7.40$ were

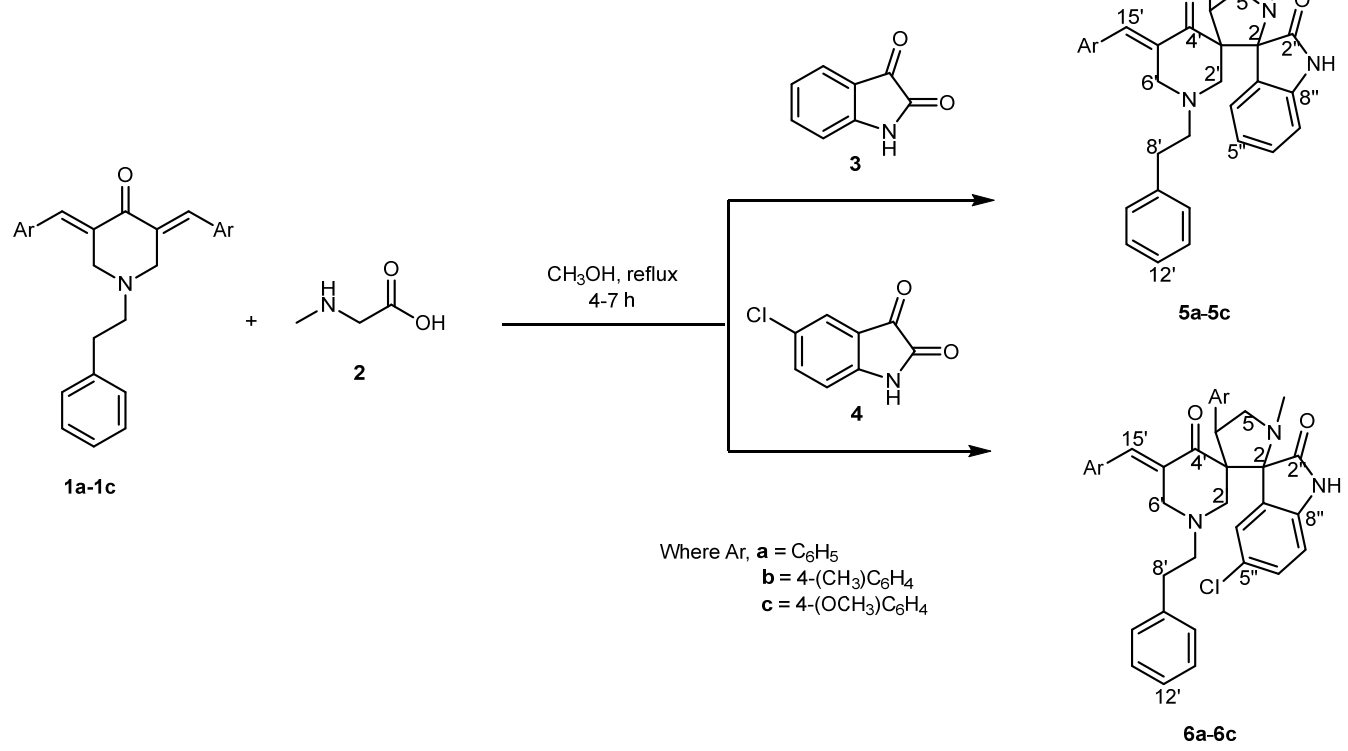

Scheme 1. Reaction scheme of synthesis of spiropyrrolidines $\mathbf{5}$ and $\mathbf{6}$ 
assigned to aromatic protons. The HSQC spectrum data of $\mathbf{6 b}$ showed all direct correlations between protons with their respective carbons in molecule $\mathbf{6 b}$. The carbon signals of $\mathrm{N}-\mathrm{CH}_{3}, \mathrm{C}-2^{\prime \prime}, \mathrm{C}-4^{\prime}$ and two methyl $\left(-\mathrm{CH}_{3}\right)$ substituent of C-28' and C-29' were assigned in ${ }^{13} \mathrm{C}-\mathrm{NMR}$ at $\delta_{\mathrm{C}} 34.6,176.7$ and 198.6, respectively. Two methyl ($\mathrm{CH}_{3}$ ) substituent of $\mathrm{CH}_{3}-28^{\prime}$ and $\mathrm{CH}_{3}-29^{\prime}$ showed a correlation with carbon signals at $\delta_{\mathrm{C}} 21.38\left(\mathrm{C}-28^{\prime}\right)$ and $21.12\left(\mathrm{C}-29^{\prime}\right)$. The signal of $\mathrm{H}-29^{\prime}$ showed correlation with $\delta_{\mathrm{C}} 20.6\left(\mathrm{C}-29^{\prime}\right), \mathrm{H}-28^{\prime}$ with $\delta_{\mathrm{C}} 20.9\left(\mathrm{C}-28^{\prime}\right), \mathrm{H}-8^{\prime}$ with $\delta_{\mathrm{C}}$ $32.0\left(\mathrm{C}-8^{\prime}\right), \mathrm{H}-4$ with $\delta_{\mathrm{C}} 44.6(\mathrm{C}-4), \mathrm{H}_{\mathrm{a}}-2^{\prime}$ and $\mathrm{H}_{\mathrm{b}}-2^{\prime} / \mathrm{H}_{\mathrm{a}}-6^{\prime}$ with $\delta_{\mathrm{C}} 56.3\left(\mathrm{C}-2^{\prime}\right), \mathrm{H}_{\mathrm{b}}-6^{\prime}$ and $\mathrm{H}_{\mathrm{b}}-2^{\prime} / \mathrm{H}_{\mathrm{a}}-6$ with $\delta_{\mathrm{C}} 56.4(\mathrm{C}-$ $\left.6^{\prime}\right)$, and $\mathrm{H}-7^{\prime}$ with $\delta_{\mathrm{C}} 59.0\left(\mathrm{C}-7^{\prime}\right)$. The peak of C-4" in $\mathbf{6 b}$ shifted slightly to downfield due to chlorine atom $(-\mathrm{Cl})$ attach at C-3". The carbon signal at $\delta_{\mathrm{C}} 54.0$ was assigned to C-5 due to its correlation with two signals of proton $\mathrm{H}_{\mathrm{a}}$ 5 and water signal. It was suggested $\mathrm{H}_{\mathrm{b}}-5$ overlapped with the water signal at $\delta_{\mathrm{C}} 3.39$. Based on the DEPT135 spectrum of $\mathbf{6 b}$, two quaternary carbons at $\delta_{\mathrm{C}} 75.2$ and 64.9 were assigned due to the spiro carbons C-2 and C-3, respectively. The carbon for the carbonyl group can be observed at a downfield of ${ }^{13} \mathrm{C}-\mathrm{NMR}$. The signals at $\delta_{\mathrm{C}}$ 176.7 and 198.6 were due to the carbonyl group and the carbonyl group of oxindole, respectively.

The HMBC spectrum data (Fig. 1) showed correlations of $\mathrm{H}-4$ with C-3, C-2', C-4', and C-aromatic. The proton of $\mathrm{N}_{-} \mathrm{CH}_{3}$ with $\mathrm{C}-3, \mathrm{C}-5$, and C-4'. These cross-correlations of proton and carbon further confirming the position of C-4, C-5, and $\mathrm{N}-\mathrm{CH}_{3}$ and two spiro carbon, $\mathrm{C}-2$, and $\mathrm{C}-3$, in the same pyrrolidine ring of compound $\mathbf{6 b}$. In addition, $\mathrm{H}-15^{\prime}$ and $\mathrm{H}-28^{\prime}$ correlation with C-17'/ C-21' and C-18'/C-20', while $\mathrm{CH}_{2}-6$ with C-15'. Similar correlations were observed between $\mathrm{H}-4^{\prime}$ and $\mathrm{H}-$ $29^{\prime}$ with C-23'/C-27' and C-24'/C-26'. These correlations confirmed the presence of the aromatic rings. The $\mathrm{H}-15^{\prime}$ was correlated with $\mathrm{C}-5^{\prime}$ and $\mathrm{C}-4$ ', thus confirming the location of the aromatic ring with piperidinone. The $\mathrm{H}$ $2^{\prime} / \mathrm{H}-6^{\prime}$ show correlation with $\mathrm{C}-2, \mathrm{C} 4^{\prime}, \mathrm{C}-3 / \mathrm{C}-5^{\prime}, \mathrm{C}-7^{\prime}$, and $\mathrm{C}-15^{\prime}$. The $\mathrm{H}-7^{\prime}$ show correlation with $\mathrm{C}-2^{\prime} / \mathrm{C}-6^{\prime}$, and C-9'. The H-8' show correlation with C-7', C-9', and Caromatic $\left(\mathrm{C}-10^{\prime}\right.$ and $\left.\mathrm{C}^{\prime} 1^{\prime}\right)$. The $\mathrm{H}$-aromatic show correlation with C-9' and C-aromatic. These correlations confirmed the aromatic ring with piperidinone ring of

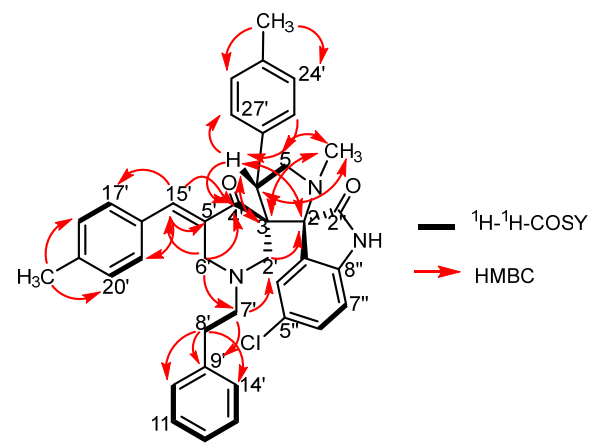

Fig 1. Selected ${ }^{1} \mathrm{H}-{ }^{1} \mathrm{H}$ COSY and $\mathrm{HMBC}$ correlations of 6b

compound 6b. The elucidated structure of the compound was further confirmed with mass spectroscopy analysis. The spectral of compounds obtained agreed with the proposed structure.

A proposed reaction mechanism for the formation of the spiro-pyrrolidine is shown in Scheme 2. The mechanism involves the formation of azomethine ylides, formed via decarboxylative condensation of isatin analogs (3 or 4 ) and sarcosine (2), which then undergo $[2+3]$ cycloaddition to give the spiro-pyrrolidines $\mathbf{5}$ and 6, respectively. Previous studies showed that spiropyrrolidine reactions are chemoselective and regioselective [26,33]. A dipole addition has occurred only to the available $\mathrm{C}=\mathrm{C}$ bond and not at the $\mathrm{C}=\mathrm{O}$ functional group of $\mathbf{1}$. The nucleophilic carbon of azomethine ylide tends to attack the end of enone fragment of the exocyclic dipolarophiles substrate $\mathbf{1}$ to produce cycloadducts $\mathbf{5}$ and $\mathbf{6}$, respectively. This reaction was conducted in methanol solvent. A previous study reported that methanol solvent has a high stabilization of polar transition state. Thus, it produces a good yield of spiro-pyrrolidine in a shorter reaction time [34]. The stereochemistry of spiropyrrolidines was compared with literature and confirmed with $\mathrm{x}$-ray structure reported by Girgis et al. [28-29].

\section{Molecular Docking Studies}

The compounds 5 and $\mathbf{6}$ were constructed using Chemdraw Professional 15.1 and were converted from sdf to pdb format before starting the docking process. The native ligand, EBN in the active site of WNV NS2BNS3 protease, was removed and re-dock $(-8.20 \mathrm{kcal} / \mathrm{mol})$ 

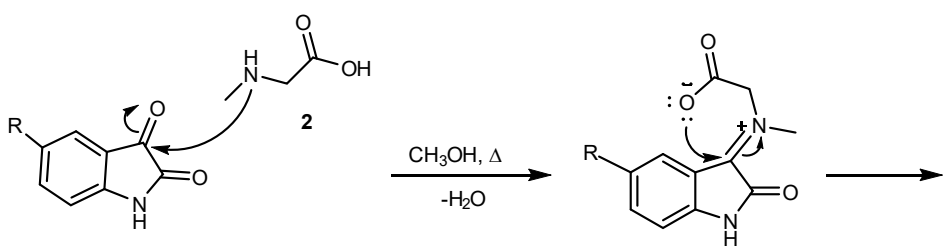<smiles>CN1CC(=O)C(=O)C12OC(=O)Nc1ccc(P)cc12</smiles>
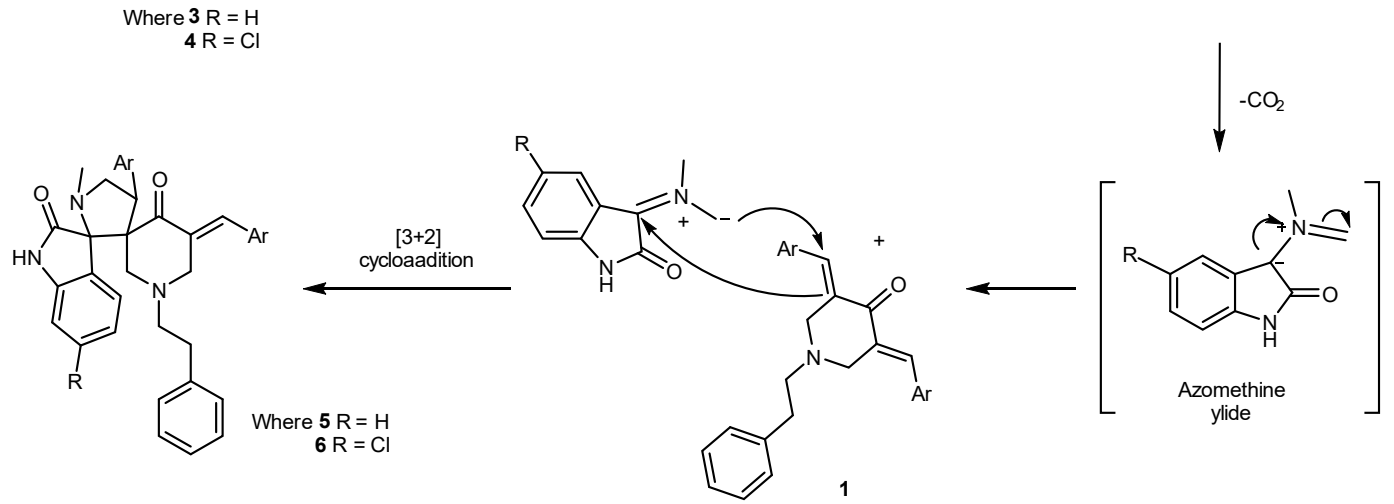

Scheme 2. Proposed mechanism for the formation of spiropyrrolidines

with the synthesized compounds using Autodock 4.2. From Table 1, it was found that all the synthesized compounds docked well into the enzyme's active site. The low binding energy indicates the formation of a favorable stable enzyme-ligand complex with a low inhibitory constant, $\mathrm{K}_{\mathrm{i}}$ value [35]. Hydrogen bonds were the primary interaction between compounds $\mathbf{5}$ and $\mathbf{6}$ with catalytic triad residue of WNV NS2B-NS3 serine protease (His51, Asp75, and Ser135) [36]. Compound 5c was postulated from the docking results to form four hydrogen bonds, while the other compounds may have two or fewer hydrogen bonds.

Based on this result, Compound $\mathbf{5 c}$ was deemed to have a good binding affinity with the enzyme with the free binding energy of $-7.71 \mathrm{kcal} / \mathrm{mol}$ and estimated inhibition constant, $\mathrm{K}_{\mathrm{i}}$ of $1.73 \mu \mathrm{M}$. Fig. 2 showed the binding orientation of compound $\mathbf{5 c}$ into the active site of WNV
NS2B-NS3 protease (PDB: 2YOL) with two hydrogen interactions (yellow dotted lines) formed between $\mathbf{5} \mathbf{c}$ and

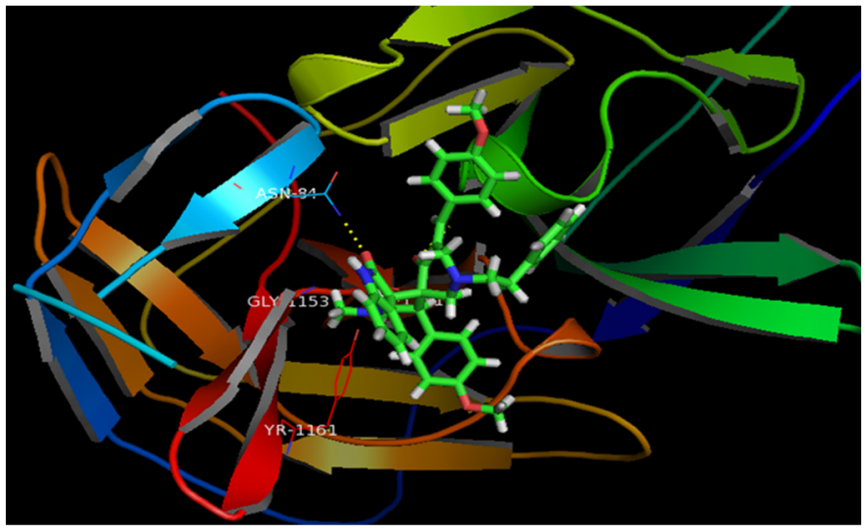

Fig 2. Docking pose of compound $\mathbf{5 c}$ in the active site of WNV NS2B-NS3 protease (PDB code: 2YOL). Hydrogen bonds which are shown as yellow dotted lines were formed between $\mathbf{5 c}$ and residues Asn84

Table 1. Binding energy and inhibitory constant of synthesized spiropyrrolidine compounds

\begin{tabular}{llccc}
\hline Compd. & Ar & $\begin{array}{c}\text { Binding energy } \\
(\mathrm{kcal} / \mathrm{mol})\end{array}$ & $\begin{array}{c}\text { Estimated inhibition } \\
\text { constant, } \mathrm{K}_{\mathrm{i}}(\mu \mathrm{m})\end{array}$ & RMSD $(\AA)$ \\
\hline 5a & Phenyl & -7.49 & 1.82 & 1.50 \\
5b & 4-Methylphenyl & -7.54 & 1.76 & 1.76 \\
5c & 4-Methoxyphenyl & -7.71 & 1.73 & 1.54 \\
6a & Phenyl & -7.56 & 1.86 & 1.82 \\
6b & 4-Methylphenyl & -7.83 & 1.71 & 1.60 \\
6c & 4-Methoxyphenyl & -7.84 & 1.83 & 1.58 \\
EBN & - & -8.20 & 0.98 & - \\
\hline
\end{tabular}




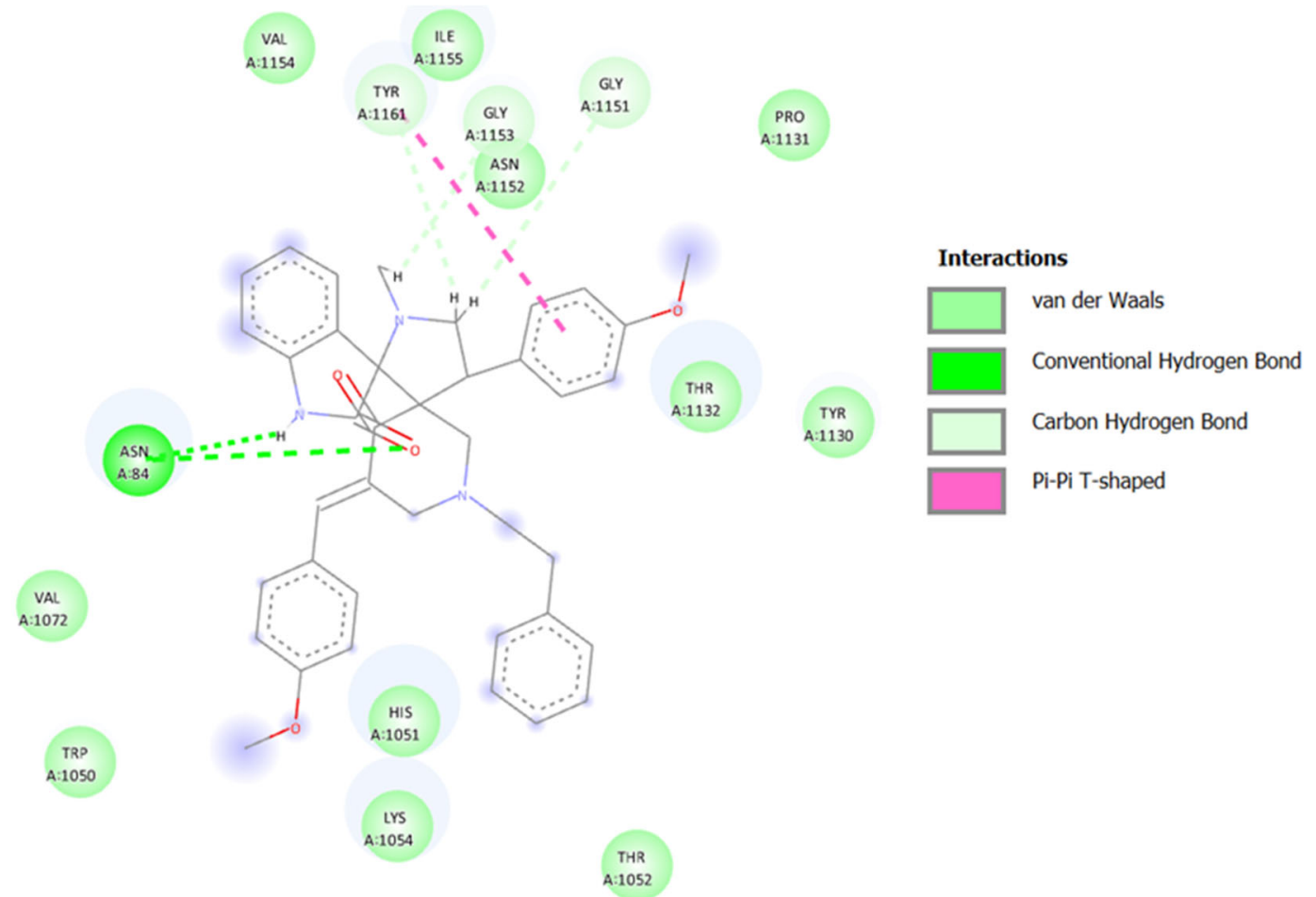

Fig 3. $2 \mathrm{D}$ interaction diagram of $\mathbf{5 c}$ with the residues within $2 \AA$ in the active pocket. Hydrogen bonding interactions were represented by green dashed lines

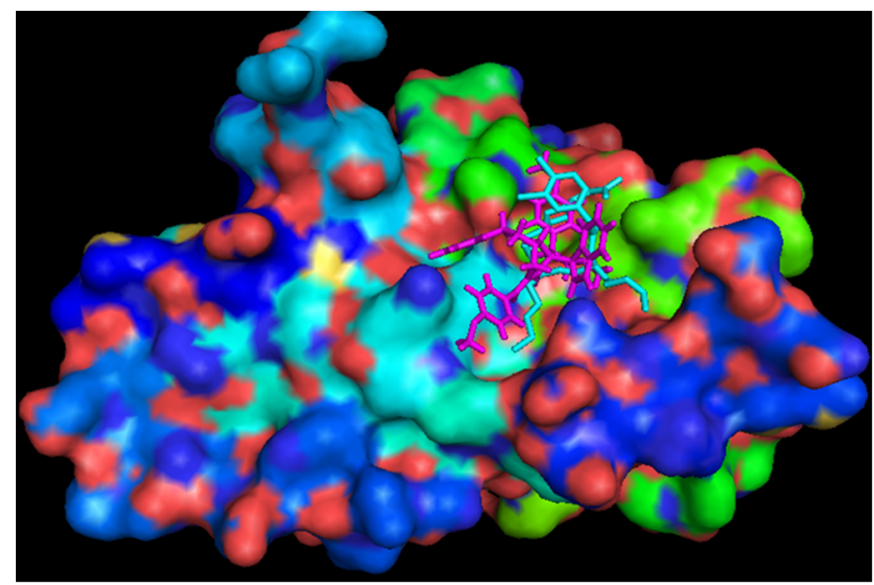

Fig 4. Comparison of docking pose of the native ligand (cyan) and $\mathbf{5 c}$ (magenta) in the active site of WNV N2BNS3 protease

residues Asn84 (2.139 ̊). The distance of hydrogen bond interaction between hydrogen and heteroatom is within the range of $2.5-3.5 \AA$, with a bond angle at $109-110^{\circ}$ [37]. $\pi-\pi$ interactions between the Tyr1161 and terminal phenyl ring and two Van der Waals interactions with the Gly1151 and Gly1153 residues were also observed. The hydrogen bond formed between $\mathbf{5 c}$ with the Asn84 through the $\mathrm{C}=\mathrm{O}$ and $-\mathrm{NH}$ of the oxindole ring (Fig. 3). However, there was no interaction has been detected between compound $\mathbf{5} \mathbf{c}$ with the catalytic triad of NS2BNS3. It was observed that the complexes are exhibiting a similar type of interactions occupying the same active site pocket compared to the reference compound of the native ligand. Fig. 4 showed docking poses of native ligand and $\mathbf{5 c}$. The structure of synthesized compounds was more complex and bulkier, thus hindering them from embedding well into the enzyme's active site. As this is a preliminary prediction, further experimental studies are needed to validate the docking results.

\section{- CONCLUSION}

The facile one-pot, three components [3+2]cycloaddition protocol leading to the synthesis of new spiro-pyrrolidines has been described. In docking studies, compound $\mathbf{5 c}$ shows a promising result as the inhibitor for WNV with four hydrogen bonding occur with active site residue, the free binding energy of $-7.71 \mathrm{kcal} / \mathrm{mol}$ and estimated inhibition constant, $\mathrm{Ki}$ of $1.73 \mu \mathrm{m}$. Although the series can be useful for developing anti- 
dengue agents, further study is required to understand the interaction, improvise, and validate the result.

\section{- ACKNOWLEDGMENTS}

Mohamed Yusoff, N. thanks to the Malaysian government for the scholarship (MyBrain15) and Universiti Sains Malaysia (USM) under grant no. [RUI 1001/PKIMIA/8011072] to financially support this research.

\section{- AUTHOR CONTRIBUTIONS}

Conceptualization, HO, MAA, YKY, and MNA; methodology, NMY, MAA, YKY, HO, MNA, and VM; software, MZH, and EEK; validation, $\mathrm{HO}$, and MNA; formal analysis, NMY, MZH, MSAG, and EEK; investigation, NMY, MSAG, MZH, EEK, and the US; resources, HO, and MNA; data curation, NMY, and MSAG; writing-original draft preparation, NMY, MSAG, $\mathrm{MZH}$, and MNA; writing-review and editing, MNA, US, EEK, and MZH; visualization, NMY, and MNA; supervision, $\mathrm{HO}$, and MNA; project administration, $\mathrm{HO}$, and MNA; funding acquisition, $\mathrm{HO}$, and MNA; All authors have read and agreed to the published version of the manuscript.

\section{- REFERENCES}

[1] Pierson, T.C., and Diamond, M.S., 2020, The continued treat of emerging flaviviruses, Nat. Microbiol., 5 (6), 796-812.

[2] Mackenzie, J.S., Gubler, D.J., and Petersen, L.R., 2004, Emerging flaviviruses: The spread and resurgence of Japanese encephalitis, West Nile and dengue viruses, Nat. Med., 10 (12), S98-S109.

[3] Lim, S.P., 2019, Dengue drug discovery: Progress, challenges and outlook, Antiviral Res., 163, 156-178.

[4] World Health Organization, 2017, West Nile virus, https://www.who.int/news-room/fact-sheets/detail/ west-nile-virus, accessed on 25 August 2021.

[5] Peterson, L.R., Brault, A.C., and Nasci, R.S., 2013, West Nile virus: Review of the literature, JAMA, 310 (3), 308-315.

[6] Richner, J.M., Gmyrek, G.B., Govero, J., Tu, Y., van der Windt, G.J.W., Metcalf, T.U., Haddad, E.K., Textor, J., Miller, M.J., and Diamond, M.S., 2015,
Age-dependent cell trafficking defects in draining lymph nodes impair adaptive immunity and control of West Nile virus infection, PLoS Pathog., 11 (7), e1005027.

[7] Yao, Y., and Montgomery, R.R., 2016, Role of immune aging in susceptibility to West Nile virus, Methods Mol. Biol., 1435, 235-247.

[8] Montgomery, R.R., 2017, Age-related alterations in immune responses to West Nile virus infection, Clin. Exp. Immunol., 187 (1), 26-34.

[9] da Silva-Júnior, E.F., and de Araújo-Júnior, J.X., 2019, Peptide derivatives as inhibitors of NS2B-NS3 protease from Dengue, West Nile, and Zika flaviviruses, Bioorg. Med. Chem., 27 (18), 3963-3978.

[10] Bakonyi, T., and Haussig, J.M., 2020, West Nile virus keeps on moving up in Europe, Eurosurveillance, 25 (46), 2001938.

[11] Luo, D., Vasudevan, S.G., and Lescar, J., 2015, The flavivirus NS2B-NS3 protease-helicase as a target for anti-viral drug development, Antiviral Res., 118, 148-158.

[12] Bastos Lima, A., Behnam, M.A.M., El Sherif, Y., Nitsche, C., Vechi, S.M., and Klein, C.D., 2015, Dual inhibitors of the dengue and West Nile virus NS2B-NS3 proteases: Synthesis, biological evaluation and docking studies of novel peptidehybrids, Bioorg. Med. Chem., 23 (17), 5748-5755.

[13] Chappell, K., Stoermer, M., Fairlie, D., and Young, P., 2008, West Nile virus NS2B/NS3 protease as anti-viral target, Curr. Med. Chem., 15 (27), 27712784.

[14] Li, Z., Sakamuru, S., Huang, R., Brecher, M., Koetzner, C.A., Zhang, J., Chen, H., Qin, C.F., Zhang, Q.Y., Zhou, J., Kramer, L.D., Xia, M., and Li, H., 2018, Erythrosin B is a potent and broadspectrum orthosteric inhibitor of the flavivirus NS2B-NS3 protease, Antiviral Res., 150, 217-225.

[15] Skoreński, M., Milewska, A., Pyrć, K., Sieńczyk, M., and Oleksyszyn, J., 2019, Phosphonate inhibitors of West Nile virus NS2B/NS3 protease, J. Enzyme Inhib. Med. Chem., 34 (1), 8-14.

[16] Kang, C., Gayen, S., Wang, W., Severin, R., Chen, A.S., Lim, H.A., Chia, C.S.B., Schüller, A., Doan, 
D.N.P., Poulsen, A., Hill, J., Vasudevan, S.G., and Keller, T.H., 2013, Exploring the binding of peptidic West Nile virus NS2B-NS3 protease inhibitors by NMR, Antiviral Res., 97 (2), 137-144.

[17] Wei, A.C., Ali, M.A., Yoon, K.Y., Ismail, R., Choon, T.S., Kumar, R.S., Arumugam, N., Almansour, A.I., and Osman, H., 2012, Antimycobacterial activity: A facile three-component [3+2]-cycloaddition for the regioselective synthesis of highly functionalised dispiropyrrolidines, Bioorg. Med. Chem. Lett., 22 (15), 4930-4933.

[18] Wei, A.C., Ali, M.A., Yoon, K.Y., Ismail, R., Choon, T.S., and Kumar, R.S., 2013, A facile threecomponent [3+2]-cycloaddition for the regioselective synthesis of highly functionalised dispiropyrrolidines acting as antimycobacterial agents, Bioorg. Med. Chem. Lett., 23 (5), 1383-1386.

[19] Wei, A.C., Ali, M.A., Yoon, K.Y., Ismail, R., Choon, T.S., Khaw, K.Y., Murugaiyah, V., and Lakshmipathi, V.S., 2014, Synthesis of highly functionalised dispiropyrrolidine derivatives as novel acetylcholinesterase inhibitors, Lett. Drug Des. Discovery, 11 (2), 156-161.

[20] Kumar, R.S., Rajesh, S.M., Perumal, S., Banerjee, D., Yogeeswari, P., and Sriram, D., 2010, Novel threecomponent domino reactions of ketones, isatin and amino acids: Synthesis and discovery of antimycobacterial activity of highly functionalised novel dispiropyrrolidines, Eur. J. Med. Chem., 45 (1), 411-422.

[21] Lawson, S., Arumugam, N., Almansour, A.I., Kumar, R.S., and Thangamani, S., 2020, Dispiropyrrolidine tethered piperidone heterocyclic hybrids with broadspectrum antifungal activity against Candida albicans and Cryptococcus neoformans, Bioorg. Chem., 100, 103865.

[22] Karthikeyan, K., Sivakumar, P.M., Doble, M., and Perumal, P.T., 2010, Synthesis, antibacterial activity evaluation and QSAR studies of novel dispiropyrrolidines, Eur. J. Med. Chem., 45 (8), 34463452.

[23] Hassaneen, H.M., Eid, E.M., Eid, H.A., Farghaly, T.A., and Mabkhot, Y.N., 2017, Facial regioselective synthesis of novel bioactive spiropyrrolidine/ pyrrolizine-oxindole derivatives via a three components reaction as potential antimicrobial agents, Molecules, 22 (3), 357.

[24] Bhaskar, G., Arun, Y., Balachandran, C., Saikumar, C., and Perumal, P.T., 2012, Synthesis of novel spirooxindole derivatives by one pot multicomponent reaction and their antimicrobial activity, Eur. J. Med. Chem., 51, 79-91.

[25] Almansour, A.I., Arumugam, N., Kumar, R.S., Althamili, D.M., Periyasami, G., Ponmurugan, K., AlDhabi, N.A., Perumal, K., and Premnath, D., 2019, Domino multicomponent approach for the synthesis of functionalized spiro-Indeno[1,2$b$ ]quinoxaline heterocyclic hybrids and their antimicrobial activity, synergistic effect and molecular docking simulation, Molecules, 24 (10), 1962-1976.

[26] Almansour, A.I., Kumar, R.S., Beevi, F., Shirazi, A.N., Osman, H., Ismail, R., Choon, T.S., Sullivan, B., McCaffrey, K., Nahhas, A., and Parang, K., 2014, Facile, regio-and diastereoselective synthesis of spiro-pyrrolidine and pyrrolizine derivatives and evaluation of their antiproliferative activities, Molecules, 19 (7), 10033-10055.

[27] Girgis, A.S., Panda, S.S., Ahmed Farag, I.S., ElShabiny, A.M., Moustafa, A.M., Ismail, N.S.M., Pillai, G.G., Panda, C.S., Hall, C.D., and Katritzky, A.R., 2015, Synthesis, and QSAR analysis of antioncological active spiro-alkaloids, Org. Biomol. Chem., 13 (6), 1741-1754.

[28] Girgis, A.S., Panda, S.S., Aziz, M.N., Steel, P.J., Hall, C.D., and Katritzky, A.R., 2015, Rational design, synthesis, and 2D-QSAR study of anti-oncological alkaloids against hepatoma and cervical carcinoma, RSC Adv., 5 (36), 28554-28569.

[29] Girgis, A.S., Panda, S.S., Shalaby, E.M., Mabied, A.F., Steel, P.J., Hall, C.D., and Katritzky, A.R., 2015, Regioselective synthesis and theoretical studies of an anti-neoplastic fluoro-substituted dispirooxindole, RSC Adv., 5 (19), 14780-14787.

[30] Murugan, R., Anbazhagan, S., and Narayanan, S.S., 2009 , Synthesis and in vivo antidiabetic activity of 
novel dispiropyrrolidines through [3+2] cycloaddition reactions with thiazolidinedione and rhodanine derivatives, Eur. J. Med. Chem., 44 (8), 3272-3279.

[31] Toumi, A., Boudriga, S., Hamden, K., Sobeh, M., Cheurfa, M., Askri, M., Knorr, M., Strohmann, C., and Brieger, L., 2021, Synthesis, antidiabetic activity and molecular docking study of rhodanine-substitued spirooxindole pyrrolidine derivatives as novel $\alpha$ amylase inhibitors, Bioorg. Chem., 106, 104507.

[32] Ali, M.A., Lakshmipathi, V.S., Beevi, F., Kumar, R.S., Ismail, R., Choon, T.S., Wei, A.C., Yoon, Y.K., and Basiri, A., 2013, Antimycobacterial activity: Synthesis and biological evaluation of novel substituted (3E,5E)-3,5-diarylidene-1-phenethylpiperidine-4-one derivatives, Lett. Drug Des. Discovery, 10 (5), 471-476.

[33] Shalaby, E.M., Girgis, A.S., Moustafa, A.M., ElShaabiny, A.M., El-Gendy, B.E.M., Mabied, A.F., and Farag, I.S.A., 2014, Regioselective synthesis, stereochemical structure, spectroscopic characterization and geometry optimization of dispiro [3H-indole-3,2'-pyrrolidine-3',3"'-piperidines], J. Mol. Struct., 1075, 327-334.
[34] Kumar, R.S., and Perumal, S., 2007, Novel threecomponent tandem reactions of cyclic mono ketones, isatin and sarcosine: Formation of dispiropyrrolidines, Tetrahedron Lett., 48 (40), 7164-7168.

[35] Osman, H., Idris, N.H., Kamarulzaman, E.E., Wahab, H.A., and Hassan, M.Z., 2017, 3,5Bis(arylidene)-4-piperidones as potential dengue protease inhibitors, Acta Pharm. Sin. B, 7 (4), 479484.

[36] Skoreński, M., Milewska, A., Pyrć, K., Sieńczyk, M., and Oleksyszyn, J., 2019, Phosphonate inhibitors of West Nile virus NS2B/NS3 protease, J. Enzyme Inhib. Med. Chem., 34(1), 8-14.

[37] de Oliveira, A.S., Gazolla, P.A.R., Oliveira, A.F.C.S., Pereira, W.L., de S Viol, L.C., Maia, A.F.S., Santos, E.G., da Silva, Í.E.P., Mendes, T.A.O., da Silva, A.M., Dias, R.S., da Silva, C.C., Polêto, M.D., Teixeira, R.R., and de Paula, S.O., 2019, Discovery of novel West Nile Virus protease inhibitor based on isobenzonafuranone and triazolic derivatives of eugenol and indan-1,3-dione scaffolds, PLoS One, 14 (9), e0223017. 